\section{Nadelstichverletzungen im Krankenhaus: „Wenn was passiert, bitte melden!“}

\author{
Das Risiko sinkt, sich bei der Arbeit im OP und auf Station mit \\ gefährlichen Infektionskrankheiten wie Hepatitis B (HBV) und C \\ (HCV) oder dem HI-Virus (HIV) anzustecken. Gründe für den \\ positiven Trend erläutert ein Experte der zuständigen Berufs- \\ genossenschaft im ZfOU-Gespräch.
}

Sie sind Leiter der Abteilung Arbeitsmedizin bei der BGW, was ist Ihre Aufgabe?

Wir betreiben Forschung und entwickeln Präventionsstrategien. So werten wir die hauseigenen Statistiken der BGW zum Unfallgeschehen aus, auch um daraus Konzepte zur Prävention zu generieren. Die Daten zu Unfällen und Berufskrankheiten gehen zunächst über die Ärzte, Arbeitgeber oder Krankenkassen bei den 11 Bezirksverwaltungen ein und werden dann in unsere gemeinsame Datenbank BKDOK (Berufskrankheiten Dokumentationssystem) eingespeist.

Was wissen Sie zu den Infektionsrisiken am Arbeitsplatz?

Da haben wir einen erfreulichen Trend, gut dokumentierte Abnahmen der Fälle. Ein wichtiger Ansteckungsfaktor bei diesem Geschehen sind bekanntlich die Nadelstichverletzungen (NSV), darunter fallen nicht nur Verletzungen an Kanülen, sondern auch an Skalpellen, generell allen scharfkantigen, schneidenden Gerätschaften, die mit Blut oder Körperflüssigkeiten kontaminiert sind. Es waren 2013 über 60000 Meldungen, diese Zahlen steigen an ( $\odot$ Abb. 1).

Wie bitte? Sie sprachen doch eben von einer erfreulichen

\section{Abnahme?} eher die Dunkelziffer geringer wird, dass einfach besser gemeldet wird. Es gibt keine Meldepflicht bei NSV und es hängt sehr vom jeweiligen Krankenhaus oder vom Pflegeheim ab, ob uns solche Vorfälle gemeldet werden oder nicht. Die Zahlen, die bei uns ankommen, sind die Fälle, in denen die Betroffenen nach einer NSV zum
Ja, denn wir gehen davon aus, dass hier
Durchgangsarzt gehen, der dann wiederum allein schon zu Abrechnungszwecken bei der Berufsgenossenschaft meldet. Wir gehen davon aus, dass Betroffene heute eben häufiger nach NSV zum Durchgangsarzt gehen.

Und wie kommen Sie auf die gute Nachricht sinkender Infektionen?

Anhand der parallel gemeldeten Berufskrankheiten. Wir orientieren uns da vor allem an den Zahlen für berufsbedingte Infektionen an HCV, HBV und an HIV. Die Zahl der Hepatitis C-Neuinfektionen bei unseren Versicherten sinkt seit Jahren (siehe Abb. 1 auf Seite 425). Im Jahr 2013 waren es gerade noch 26 neu anerkannte HCV-Infektionen als Berufskrankheiten in unserer Datenbank, verglichen mit 103 im Jahr 2000. Das Geschehen bei HCV ist bei uns der wichtigste Indikator für berufsbedingte Infektionen. Das nimmt ab.

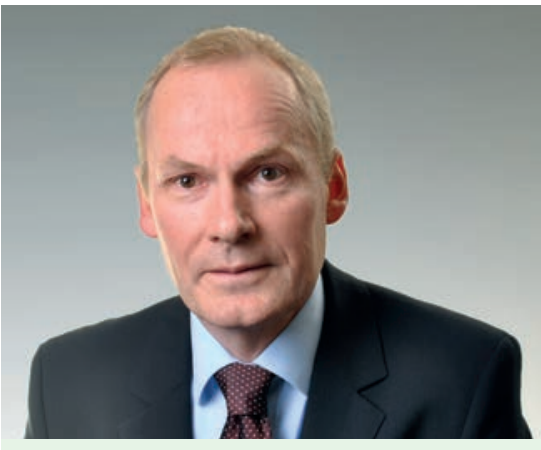

Prof. Dr. med. Albert Nienhaus ist Leiter der Abteilung Arbeitsmedizin, Gesundheitswissenschaften und Gefahrstoffe bei der Berufsgenossenschaft für Gesundheitsdienst und Wohlfahrtspflege (BCW) in Hamburg, bei der rund 8 Millionen Beschäftigte gegen Unfälle versichert sind, darunter über 2,6 Millionen Ärzte und Krankenpfleger. Außerdem führt er das Kompetenzzentrum für Epidemiologie und Versorgungsforschung bei Pflegeberufen (CVcare) am Universitätsklinikum HamburgEppendorf.

\section{Schaut man auf die Anste-} ckungswege für HCV generell in Deutschland, dann fallen die berufsbedingten Fälle kaum ins Gewicht.

So ist es, 2013 waren es 5156 Neuinfektionen an HCV und die meisten Menschen stecken sich nach wie vor beim Drogenkonsum an, am Gebrauch kontaminierter Spritzen - das zeigen die Zahlen für den Teil der Infektionen, wo der Ansteckungspfad beschrieben werden kann.

Wie unterscheiden Sie eine Neuinfektion etwa an HCV, die wirklich eine Berufskrankheit ist, da sich ein Arzt an einer infektiösen Nadel gestochen hat, mit der er zu-

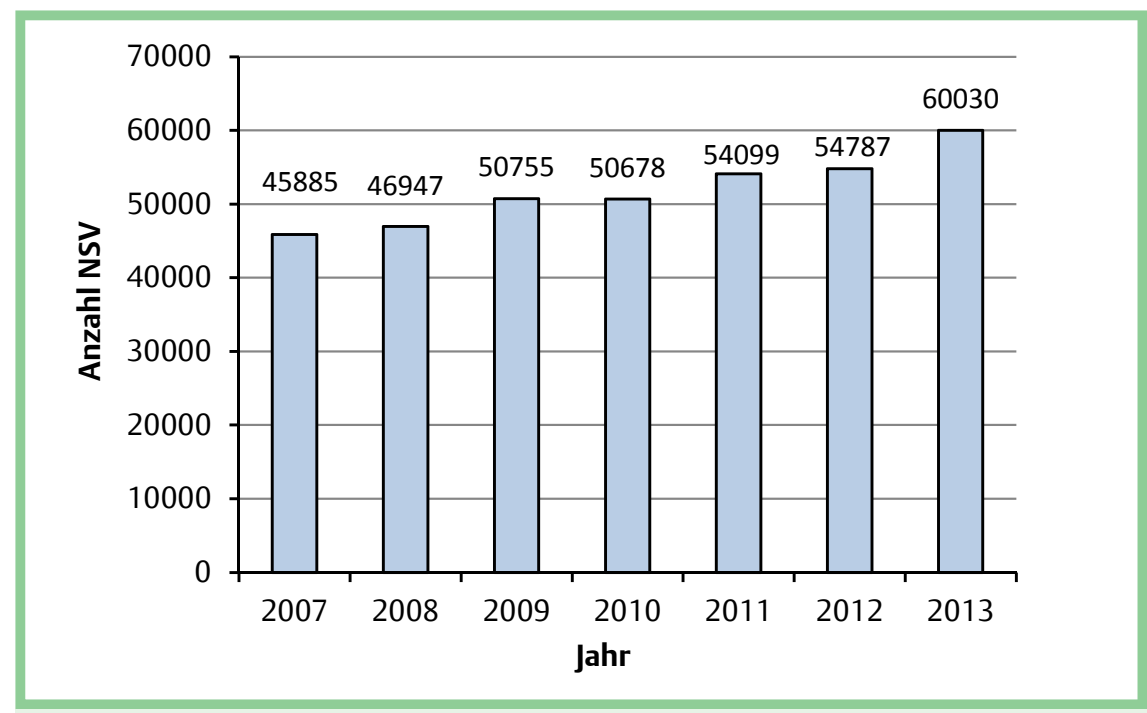

Abb. 1 Anzahl der bei der BGW gemeldeten Nadelstichverletzungen (NSV) zwischen 2007-2013. 
vor dem Patienten Blut abgenommen hat, von einem Fall, wo ein Beschäftigter zwar beim Betriebsarzt neu die Diagnose HCV erfährt, sich aber womöglich ganz woanders angesteckt hat.

Das ist durchaus schwierig. Allein schon unsere BGW-Zahl an neu anerkannten Berufskrankheiten an HCV-Infektionen im Jahr hat mehrere Teilmengen. Wir hatten im Jahr 2013 nur 5 Fälle einer HCV-Neuinfektion, in denen ein unmittelbarer $\mathrm{Zu}$ sammenhang mit einer NSV gegeben war. Bei den anderen 21 Fällen wurde ein historischer Kontext zum Beruf ermittelt. Das ist etwa, wenn bei einer Krankenpflegerin in der Nachbetrachtung eruiert werden kann, dass sie viele Jahre in Patientenkontakt war und Blutentnahmen oder Injektionen vorgenommen hat. Der zeitliche Zusammenhang mit der NSV ist dann sehr lose, dennoch kann auch so eine Berufskrankheit unter Umständen anerkannt werden.

Die Abnahme an Neuinfektionen gilt zunächst Mal für das Geschehen, das Sie als BGW überblicken. Nach Zahlen der DGUV (Deutsche Gesetzliche Unfallversicherung) wurden im Jahr 201434 neue Berufskrankheiten einer HCVInfektionen bei Beschäftigten im Gesundheitswesen in Deutschland insgesamt anerkannt.

Ja, dort sind auch die Zahlen mit dabei, die über die Unfallkassen kommen, bei denen Ärzte und Pfleger versichert sind, die an öffentlichen Kliniken arbeiten. Ein Abnahmetrend über die letzten Jahre ist auch dort erkennbar.

Auch einige bundesdeutsche Zusammenstellungen gehen in die gleiche Richtung.

So ist es. Wir gehen davon aus, dass die Zahl derer im Gesundheitswesen, die sich jährlich noch neu im Beruf an HBV, HCV oder HIV anstecken, deutlich unter 100 liegt, Tendenz fallend.

\section{Woher kommt der Erfolg?}

Wir gehen einmal davon aus, dass es heute eben weniger NSV gibt als noch vor Jahren. Eine Rolle dürften dabei die sicheren Instrumente spielen, die heute gemäß den Vorschriften der TRBA 250 in vielen Bereichen im Krankenhaus und in den Arztpraxen Pflicht sind. Zugleich steigt in dem Moment, wo ich sicherere Instrumente verwende, wo ich Schulungen dazu mache, auch das Bewusstsein für das The- ma in einem Haus - damit die Sensibilität für dieses Thema bei den Mitarbeitern. Vom Prinzip her kann man sagen, dass diese sicheren Systeme immer dann zum Einsatz kommen müssen, wenn es hektisch werden könnte, auch wenn sie teurer sind als herkömmliche.

\section{Also zum Beispiel im Schock- raum?}

Zwingend, ja. Auch bei den Skalpellen gibt es stumpfe Skalpelle, bei denen das Verletzungsrisiko geringer wird.

\section{Wer ist dafür zuständig, dass} die überall Anwendung finden, wo es vorgeschrieben ist?

Das ist klar die Verantwortung der Leitung einer Klinik. Der Arbeitgeber muss im Zweifel zumindest nachweisen können, dass er das Schutzniveau der TRBA 250 erreicht, auch dann, wenn er im Detail von den dortigen Regeln abweicht. Der Beschäftigte ist in jedem Fall bei uns versichert, es kann aber sein, dass die BG sich Auslagen vom Arbeitgeber zurückholt sollte sich zeigen, dass Sicherheitsvorschriften grob fahrlässig nicht umgesetzt wurden.

\section{Kommt das vor?}

Selten, es gibt Einzelfälle.

Haben Sie auch eine Kontrollaufgabe in Kliniken, ob die Vorschriften eingehalten werden? Nein, das ist nicht die Aufgabe meiner Abteilung. Es gibt 11 verschiedene Bezirksverwaltungen der BGW in Deutschland, in denen Präventionsdienstleister arbeiten. Deren Aufgabe ist die Beratung und Kontrolle vor Ort in den Kliniken.

Wie hoch ist das Infektionsrisiko konkret? Angenommen, mit der Kanüle wurde gerade tatsächlich einem infizierten Patienten Blut abgenommen, werde ich mich immer infizieren, sollte ich mich selber damit verletzt?

Nein. Dazu gibt es Schätzungen aus der Literatur. Bei HBV geht man von einem Infektionsrisiko von etwa $30 \%$ aus, bei HCV von $3 \%$ und bei HIV noch von $0,3 \%$ - sollte der Indexpatient tatsächlich infiziert sein. Das sind nur grobe Abschätzungen. Außerdem ist natürlich nur ein Teil der Patienten infiziert. Nach den Zahlen des RKI (Robert Koch-Institut) sind das bei HBV und HCV etwa 6,0\% der Bevölkerung, bei HIV $0,05 \%$. Wenn Sie das auf die Zahl von etwa 60000 NSV beziehen, die wir 2013 gemeldet bekamen, können Sie überschlagen, dass es im Jahr 201327 frische Infekte an HBV und HCV, sowie HIV wegen NSV gegeben haben müsste. In der Statistik hatten wir aber nur die eben schon erwähnten 5 Fälle einer Neuinfektion an HCV aufgrund einer NSV. Keine Meldung mit HBV und NSV und keinen Fall mit HIVNeuinfektion. All dies deutet auf eine Verringerung der Risiken.

Was hat die 22 zu erwartenden Neuinfektionen verhindert, die Sie nicht in der Datenbank finden?

Ein Teil geht vermutlich auf Erfolge der heute besseren Prophylaxe nach einer Verletzung zurück. Daher zunächst mal unser Appell: wenn was passiert ist, bitte zum Betriebsarzt und bitte melden! Das erleichtert auch die spätere Anerkennung einer Berufskrankheit. Und je früher eine Infektion festgestellt wird, desto besser kann man behandeln.

Was kann man nach einer NSV medizinisch unternehmen? Man wird einmal den Impfstatus gegen HBV überprüfen. Dort haben wir ja zum Glück heute die Möglichkeit zur Impfung. Auch wenn ein Arzt noch nicht geimpft ist, kann die Impfung unmittelbar nach einer Verletzung noch helfen. Langfristig sollte Hepatitis B eigentlich gar kein Problem mehr sein, denn jeder sollte sich impfen lassen.

\section{Ist diese Impfung Pflicht für} Beschäftigte im Gesundheitswesen?

Nein, in Deutschland gibt es keine Impfpflicht. Andererseits sollte ein Chirurg, der nicht gegen HBV geimpft ist, meiner Meinung nach eigentlich erst gar nicht in den OP. Und es gibt jetzt nach dem Infektionsschutzgesetz für Arbeitgeber im Gesundheitswesen die Möglichkeit, dass sie das Recht haben, sich nach dem Impfstatus von Bewerbern zu erkundigen.

Und wenn der neu anzustellende Arzt dann sagt, er hat keine HBV-Impfung und er will auch keine?

Dann läuft er das Risiko, den Job nicht zu bekommen. Das kann der Arbeitgeber für alle Impfungen, die die STIKO (Ständige Impfkommission) empfiehlt, so festlegen. Das erhöht ein bisschen den Druck, sich impfen zu lassen und damit die Sicherheit für Mitarbeiter wie Patienten im Krankenhaus. 


\section{Bei HCV gibt es keine Impfung} ...

Aber es gibt seit heute sehr gute medikamentöse Therapien mit fast $100 \%$ Erfolgsquote. Und je früher diese Behandlung nach einer Infektion beginnt, desto besser - ein weiteres Argument, nach einer NSV sofort zum Arzt zu gehen.

In einem besonders unangenehmen Fall habe ich mich an einer Kanüle gestochen, mit der ich zuvor einen Patienten behandelt habe, der HIV hat. Was dann?

Auch dann wird man das engmaschig nachkontrollieren und im Zweifel sofort mit einer medikamentösen antiretroviralen Therapie als Prophylaxe beginnen. All diese Maßnahmen nennen wir Regeluntersuchungsprogramm nach NSV - wir glauben, dass ein Teil des Rückgangs der Infektionsfälle daran liegt, dass diese medizinische Nachsorge heute viel besser ist.

Eine Unsicherheit entsteht ja auch dadurch, dass man oft nicht weiß, ob der Patient nun überhaupt infektiös ist oder nicht.
Hat man dann das Recht, ihn zu testen?

Wenn er sein Einverständnis gibt, ja. Man sollte es unbedingt versuchen. Das ist eine absolut wichtige Information, um nach einer NSV gut planen zu können.

Einige wenige Kliniken testen vor allem Notfallpatienten vor der Operation routinemäßig auf HCV, HBV und HIV. Dies mit dem Argument, dass sich Ärzte und Pfleger dann im Zweifel bei Infizierten besser mit Schutzmaßnahmen und Prophylaxe darauf einstellen können. Sinnvoll oder angesichts der von Ihnen skizzierten Abnahme der Risiken eher ein bisschen Overkill? Ich finde so etwas prinzipiell gut, denn das hat die Chance, Risiken weiter zu minimieren. Jeder Fall einer berufsbedingten Infektion, der auftritt, ist ein Fall zu viel.

Darf ein Arzt, darf ein Krankenpfleger, bei dem eine Infektion mit HBV, HCV oder HIV diagnostiziert wird, weiter arbeiten?
Ja, wobei das von einer Reihe von Faktoren abhängt. Es geht darum, die Virenlast medikamentös maximal zu senken. Bei einer hohen Virenlast, dürfte jemand bestimmte Tätigkeiten nicht mehr ausüben.

\section{Also nicht in den OP?}

Nein. So lange dürfte ein Chirurg mit HCV oder HBV nicht operieren, generell keine Dinge am Patienten verrichten. Andererseits kann auch ein Arzt mit HIV heute in den OP, wenn er seine Medikamente konstant nimmt und die Virenlast quasi unter die Nachweisgrenze sinkt.

\section{Werden die Infektionsrisiken} für Beschäftigte im Krankenhaus weiter sinken?

Ja. Seit etwa 2 Jahren kann man sagen, dass eine HCV heilbar ist. Damit wird auch dieses Problem generell abnehmen, vielleicht gar weitgehend verschwinden. Bei HBV haben wir die Impfung. Positiv sehen wir auch die Chancen gegen HIV. Die Zahlen sinken und werden weiter sinken.

Das Interview führte Bernhard Epping. 